\title{
Psoriatic Alopecia in a Patient With Crohn Disease: An Uncommon Manifestation of Tumor Necrosis Factor $\alpha$ Inhibitors
}

\author{
Osward Y. Carrasquillo, MD, MPH; Gabriela Pabón-Cartagena, MD; Julián Barrera-Llaurador, MD; \\ Francisco Colón-Fontanez, MD; Rafael F. Martín-García, MD
}

\section{PRACTICE POINTS}

- Psoriatic alopecia is a rare nonscarring alopecia that can present as a complication of treatment with tumor necrosis factor $\alpha$ inhibitors.

- This finding commonly is seen in females undergoing treatment with infliximab or adalimumab, usually for Crohn disease.

- Histopathologic findings can show a psoriasiformpattern, neutrophil-rich, inflammatory infiltrate involving hair follicles or a lichenoid pattern.

Tumor necrosis factor $\alpha$ (TNF- $\alpha$ ) inhibitors are used to treat multiple inflammatory diseases including rheumatoid arthritis, inflammatory bowel disease, and psoriasis, among others. This family of medications can cause various side effects, some as common as injectionsite reactions and others as rare as the paradoxical induction of psoriasiform skin lesions. Alopecic plaques recently have been described as an uncommon adverse effect of the TNF- $\alpha$ inhibitors adalimumab and infliximab. We present the case of a 12-year-old girl treated with adalimumab for Crohn disease who developed an alopecic crusted plaque on the scalp 6 months after increasing the dose of the medication. Biopsies, special stains, and sterile cultures yielded a diagnosis of psoriatic alopecia secondary to TNF- $\alpha$ inhibitor. A literature review for similar cases found 24 additional patients presenting with similar findings, of which only 6 were part of the pediatric population.

Cutis. 2021;107:E48-E55.

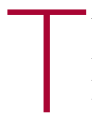
umor necrosis factor $\alpha$ (TNF- $\alpha$ ) inhibitor-induced psoriasis is a known paradoxical adverse effect of this family of medications, which includes infliximab, adalimumab, etanercept, golimumab, and certolizumab. In the pediatric population, these therapies recently gained approval for nondermatologic conditions-meaning that this phenomenon is encountered more frequently. ${ }^{1}$ In a systematic review of TNF- $\alpha$ inhibitor-induced psoriasis, severe scalp involvement was associated with alopecia in $7.5 \%$ of cases. ${ }^{2}$ Onset of scalp psoriasis with alopecia in patients being treated with a TNF- $\alpha$ inhibitor should lead to consideration of this condition.

Psoriatic alopecia is an uncommon presentation of psoriasis. Although well described, alopecia as a clinical manifestation of scalp psoriasis is not a well-known concept among clinicians and has never been widely accepted. Adding to the diagnostic challenge is that psoriatic alopecia secondary to TNF- $\alpha$ inhibitor-induced psoriasis rarely has been reported in adults or children. ${ }^{3-5}$ Including our case, our review of the literature yielded 7 pediatric cases ( $\leq 18$ years) of TNF- $\alpha$ inhibitor-induced psoriatic alopecia. ${ }^{6,7} \mathrm{~A}$ primary literature search of PubMed articles indexed for MEDLINE was conducted using the terms psoriatic alopecia, psoriasiform alopecia, TNF- $\alpha$ inhibitors, infliximab, adalimumab, etanercept, golimumab, and certolizumab.

We present the case of a pediatric patient with psoriatic alopecia secondary to treatment with adalimumab for

From the University of Puerto Rico School of Medicine, San Juan. Drs. Carrasquillo, Barrera-Llaurador, Colón-Fontanez, and Martín-García are from the Department of Dermatology, and Dr. Pabón-Cartagena is from the Transitional Year Program.

The authors report no conflict of interest.

Correspondence: Osward Y. Carrasquillo, MD, MPH, University of Puerto Rico School of Medicine, PO Box 365067, San Juan, PR 00936-5067 (ocarrasquillo5@gmail.com).

doi:10.12788/cutis.0256 
Crohn disease (CD). We also provide a review of reported cases of psoriatic alopecia induced by a TNF- $\alpha$ inhibitor in the literature.

\section{Case Report}

A 12-year-old girl presented to our dermatology clinic with erythematous scaly plaques on the trunk, scalp, arms, and legs of 2 months'duration. The lesions involved approximately $15 \%$ of the body surface area. The patient's medical history was remarkable for $\mathrm{CD}$ diagnosed 4 years prior to presentation of the skin lesions. She had been treated for the past 2 years with adalimumab $40 \mathrm{mg}$ once every 2 weeks and azathioprine $100 \mathrm{mg}$ once daily. Because her CD was poorly controlled, the dosage of adalimumab was increased to $40 \mathrm{mg}$ once weekly 6 months prior to the current presentation.

Our diagnosis was TNF- $\alpha$ inhibitor-induced psoriasis secondary to treatment with adalimumab.

The patient was treated with mometasone lotion $0.1 \%$ for the scalp lesions and triamcinolone cream $0.1 \%$ for the body lesions. Because of the extent of the psoriasis, we recommended changing adalimumab to ustekinumab, which is approved for CD in adults but is off label in children.

At 1-month follow-up, after receiving the induction dose of ustekinumab, the patient presented with partial improvement of the skin lesions but had developed a large, alopecic, erythematous plaque with thick yellowish scales on the scalp (Figure 1). She also had a positive hair pull test. The presumptive initial diagnosis of the alopecic scalp lesion was tinea capitis, for which multiple potassium hydroxide preparations of scales were performed,

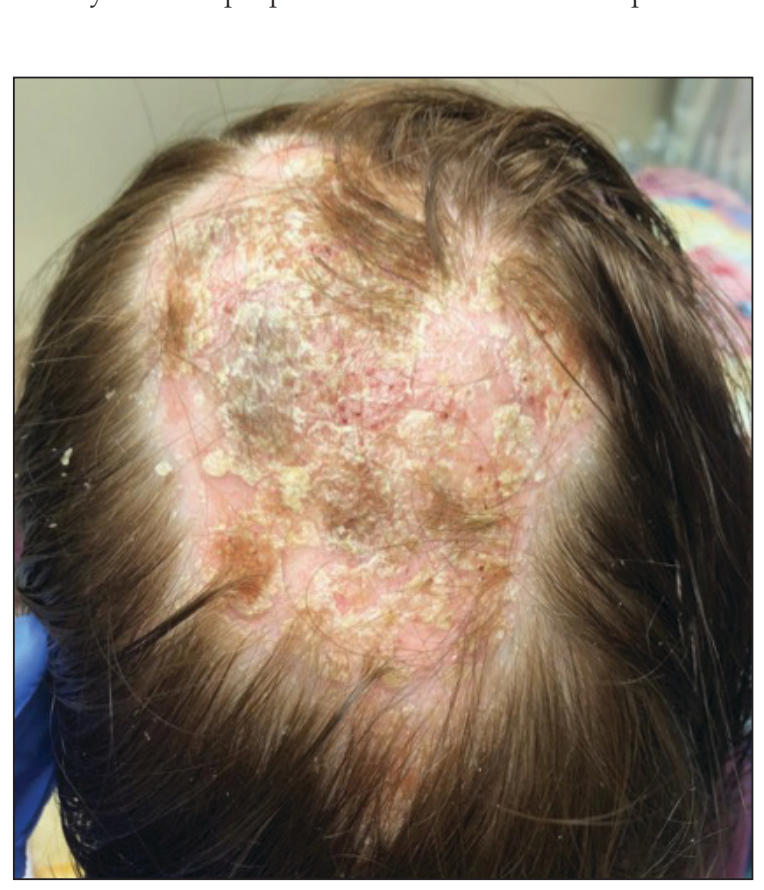

FIGURE 1. Large, alopecic, erythematous plaque on the scalp with yellowish scales. all yielding negative results. In addition, histopathologic examination with hematoxylin and eosin staining was performed (Figures 2A and 2B). Sterile tissue cultures for bacteria, fungi, and acid-fast bacilli were obtained and showed no growth. Periodic acid-Schiff staining was negative for fungal structures.

A second biopsy showed a psoriasiform pattern, parakeratosis, and hypogranulosis, highly suggestive of psoriasis (Figure 2C and 2D). Based on those findings, a diagnosis of psoriatic alopecia was made. The mometasone was switched to clobetasol lotion $0.05 \%$. The patient continued treatment with ustekinumab. At 6-month follow-up, her CD was well controlled and she showed hair regrowth in previously alopecic areas (Figure 3).

\section{Comment}

Psoriatic alopecia induced by a TNF- $\alpha$ inhibitor was first reported in 2007 in a 30-year-old woman with ankylosing spondylitis who was being treated with adalimumab. ${ }^{8}$ She had erythematous, scaly, alopecic plaques on the scalp and palmoplantar pustulosis. Findings on skin biopsy were compatible with psoriasis. The patient's severe scalp psoriasis failed to respond to topical steroid treatment and adalimumab cessation. The extensive hair loss responded to cyclosporine $3 \mathrm{mg} / \mathrm{kg}$ daily. ${ }^{8}$

After conducting an extensive literature review, we found 26 cases of TNF- $\alpha$-induced psoriatic alopecia, including the current case (Table). ${ }^{6-16}$ The mean age at diagnosis was 27.8 years (SD, 13.6 years; range, 7-60 years). The female-to-male ratio was 3.3:1. The most common underlying condition for which TNF- $\alpha$ inhibitors were prescribed was CD (77\% [20/26]). Psoriatic alopecia most commonly was reported secondary to treatment with infliximab (54\% [14/26]), followed by adalimumab (42\% [11/26]). Golimumab was the causative drug in $1(4 \%)$ case. We did not find reports of etanercept or certolizumab having induced this manifestation. The onset of the scalp lesions occurred 2 to 46 months after starting treatment with the causative medication.

Laga et $\mathrm{al}^{17}$ reported that TNF- $\alpha$ inhibitor-induced psoriasis can have a variety of histopathologic findings, including typical findings of various stages of psoriasis, a lichenoid pattern mimicking remnants of lichen planus, and sterile pustular folliculitis. Our patient's 2 scalp biopsies demonstrated results consistent with findings reported by Laga et al. ${ }^{17}$ In the first biopsy, findings were consistent with a dense neutrophilic infiltrate with negative sterile cultures and negative periodic acid-Schiff stain (sterile folliculitis), with crust and areas of parakeratosis. The second biopsy demonstrated psoriasiform hyperplasia, parakeratosis, and an absent granular layer, all typical features of psoriasis (Figure 2).

Including the current case, our review of the literature yielded 7 pediatric (ie, $0-18$ years of age) cases of TNF- $\alpha$ inhibitor-induced psoriatic alopecia. Of the 6 previously reported pediatric cases, 5 occurred after administration of infliximab. ${ }^{6,7}$ 

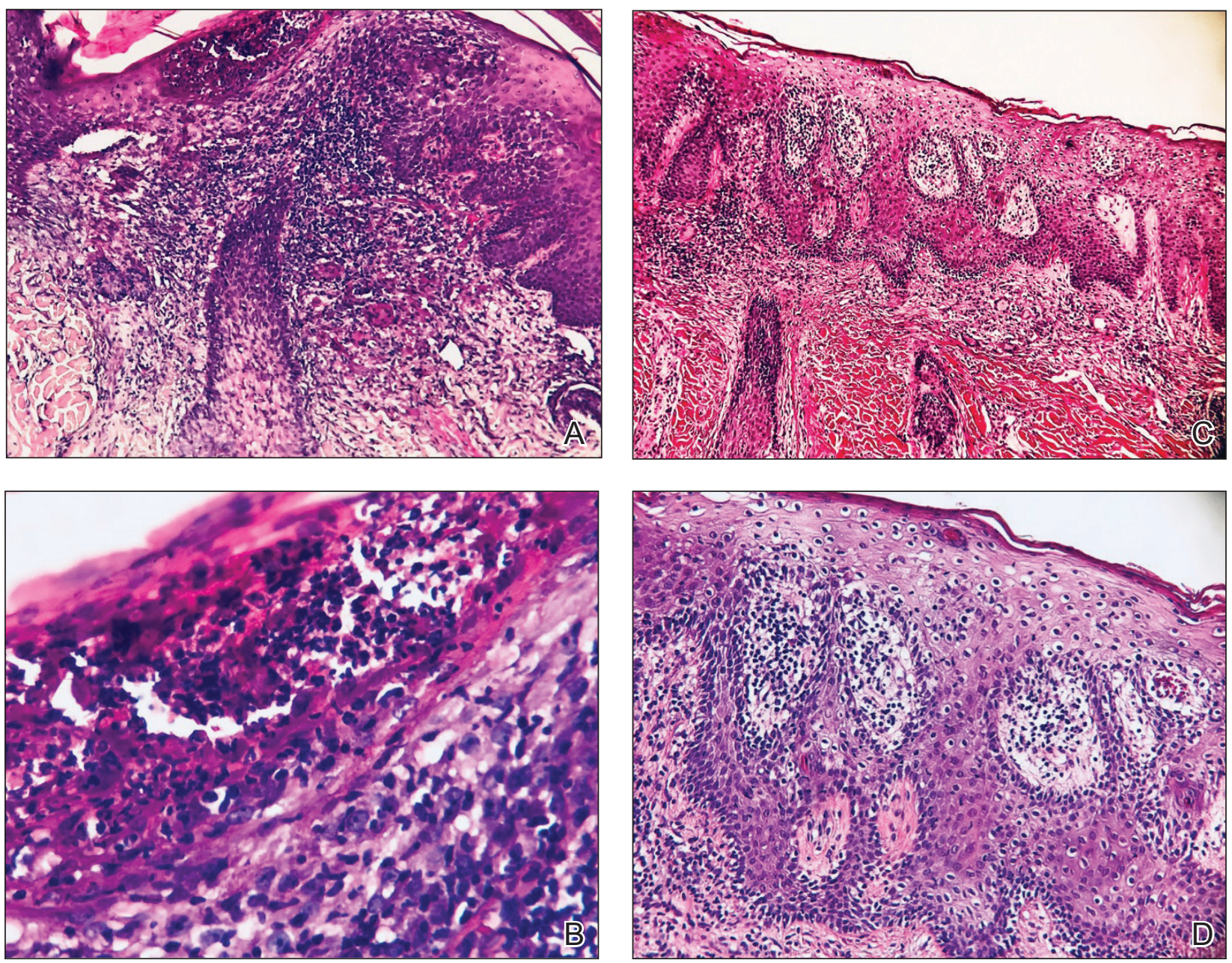

FIGURE 2. A, The first scalp biopsy showed a dense infiltrate of neutrophils in the dermis and perifollicular area (H\&E, original magnification $\times 200)$. B, The second scalp biopsy showed an intracorneal collection of neutrophils on high magnification (H\&E, original magnification $\times 400)$. $\mathrm{C}$ and $\mathrm{D}$, Psoriasiform hyperplasia with mild parakeratosis and absent granular layer on the second biopsy (H\&E, original magnifications $\times 100$ and $\times 200$ ).

Similar to our case, TNF- $\alpha$ inhibitor-induced psoriatic alopecia was reported in a 7 -year-old girl who was treated with adalimumab for juvenile idiopathic arthritis. ${ }^{6}$ Nine months after starting treatment, that patient presented with a tender, erythematous, eroded, and crusted alopecic plaque along with scaly plaques on the scalp. Adalimumab was discontinued, and cyclosporine and topical steroids were started. Cyclosporine was then discontinued due to partial resolution of the psoriasis; the patient was started on abatacept, with persistence of the psoriasis and alopecia. The patient was then started on oral methotrexate $12.5 \mathrm{mg}$ once weekly with moderate improvement and mild to moderate exacerbations.

Tumor necrosis factor $\alpha$ inhibitor-induced psoriasis may occur as a result of a cytokine imbalance. A TNF- $\alpha$ blockade leads to upregulation of interferon $\alpha$ (IFN- $\alpha$ ) and TNF- $\alpha$ production by plasmacytoid dendritic cells (pDCs), usually in genetically susceptible people. ${ }^{6,7,9-15}$
The IFN- $\alpha$ induces maturation of myeloid dendritic cells (mDCs) responsible for increasing proinflammatory cytokines that contribute to psoriasis. ${ }^{11}$ Generation of TNF- $\alpha$ by pDCs leads to mature or activated dendritic cells derived from $\mathrm{pDC}$ through autocrine TNF- $\alpha$ production and paracrine IFN- $\alpha$ production from immature mDCs. ${ }^{9}$ Once pDCs mature, they are incapable of producing IFN- $\alpha$; TNF- $\alpha$ then inhibits IFN- $\alpha$ production by inducing pDC maturation. ${ }^{11}$ Overproduction of IFN- $\alpha$ during TNF- $\alpha$ inhibition induces expression of the chemokine receptor CXCR3 on $\mathrm{T}$ cells, which recruits $\mathrm{T}$ cells to the dermis. The T cells then produce TNF- $\alpha$, causing psoriatic skin lesions. ${ }^{10,11,13,14}$

Although TNF- $\alpha$ inhibitor-induced psoriatic alopecia is uncommon, the condition should be considered in female patients with underlying proinflammatory disease$\mathrm{CD}$ in particular. Perman et $\mathrm{al}^{6}$ reported 5 cases of psoriatic alopecia in which 3 patients initially were treated with griseofulvin because of suspected tinea capitis. 


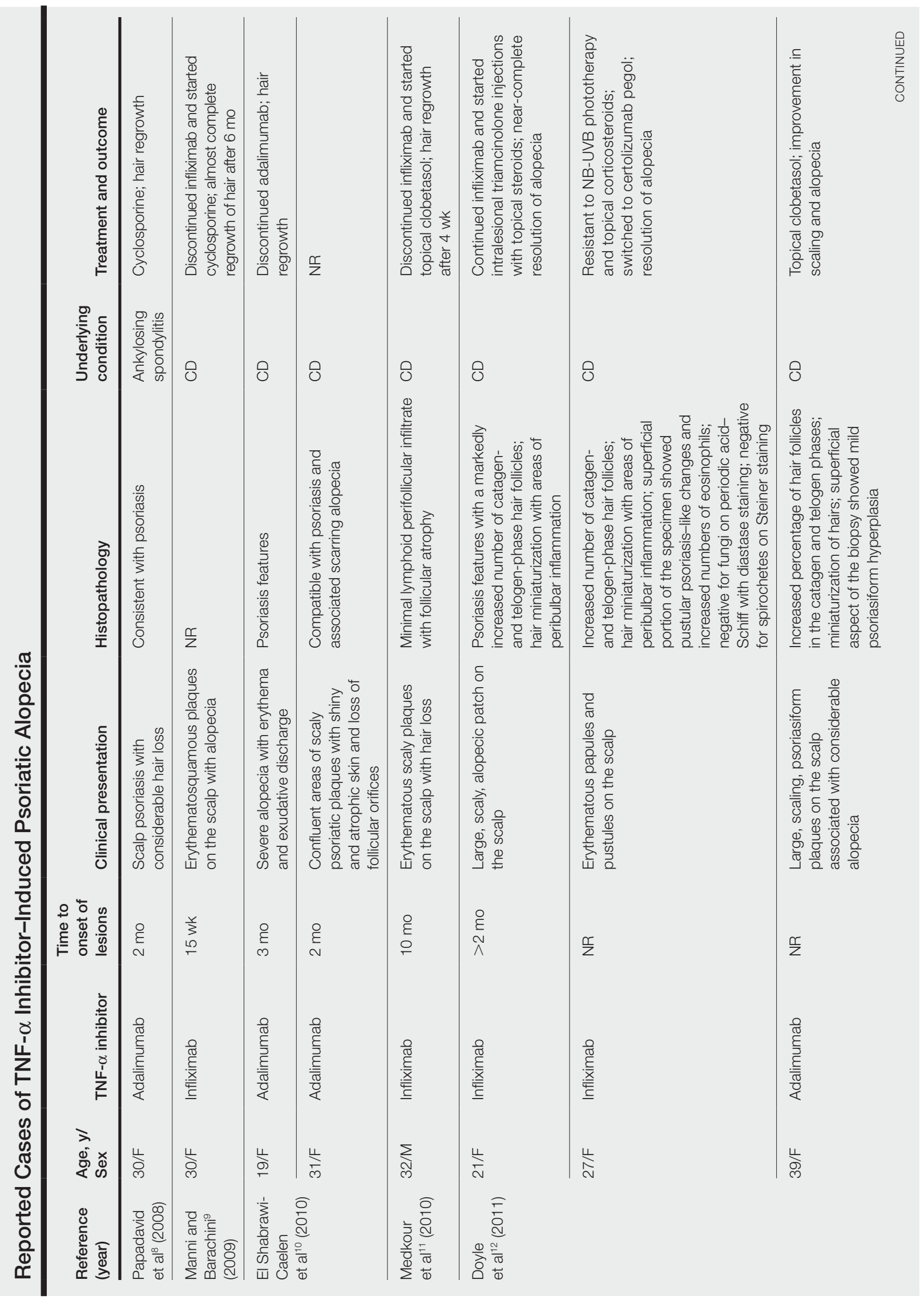




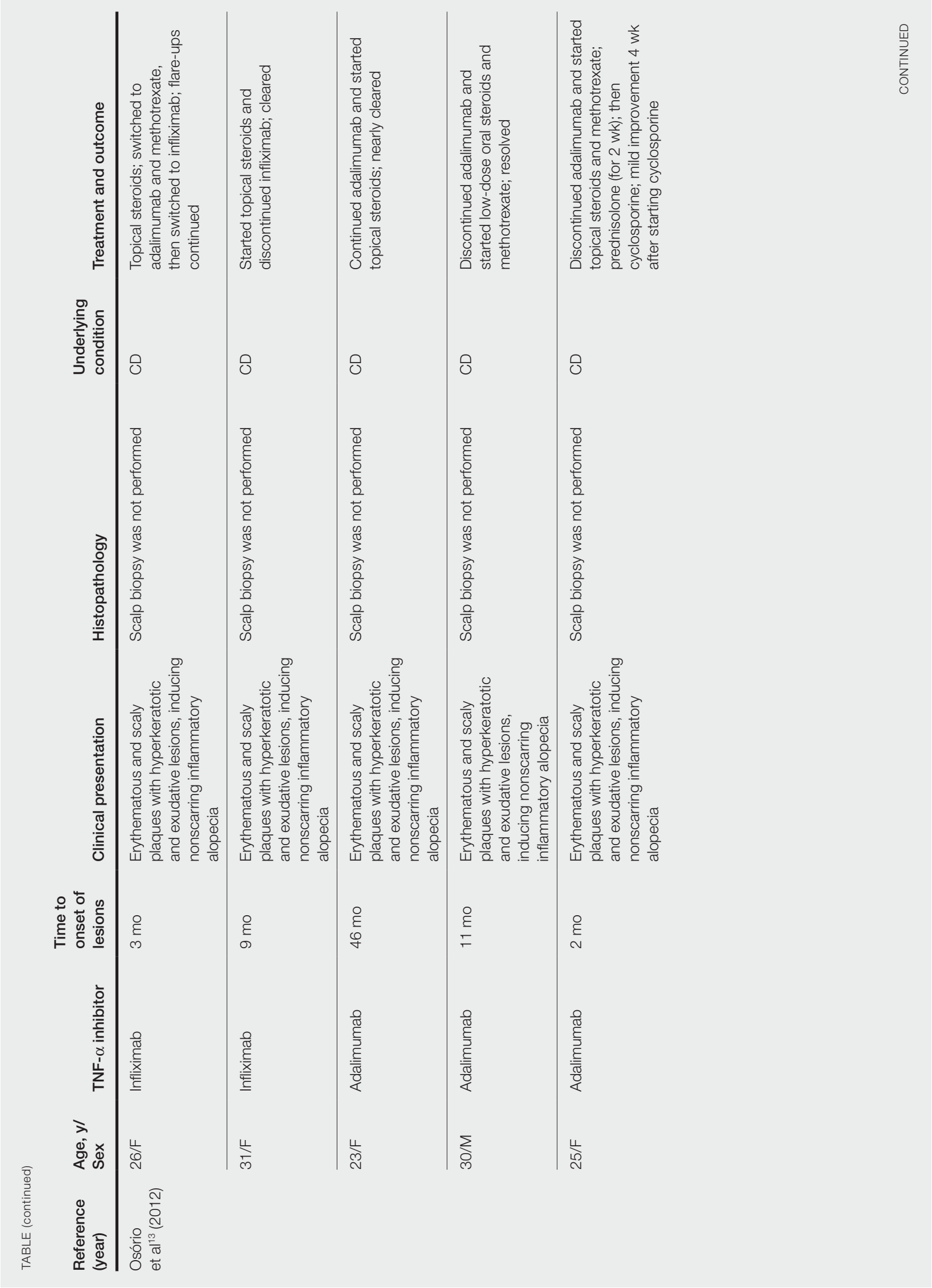




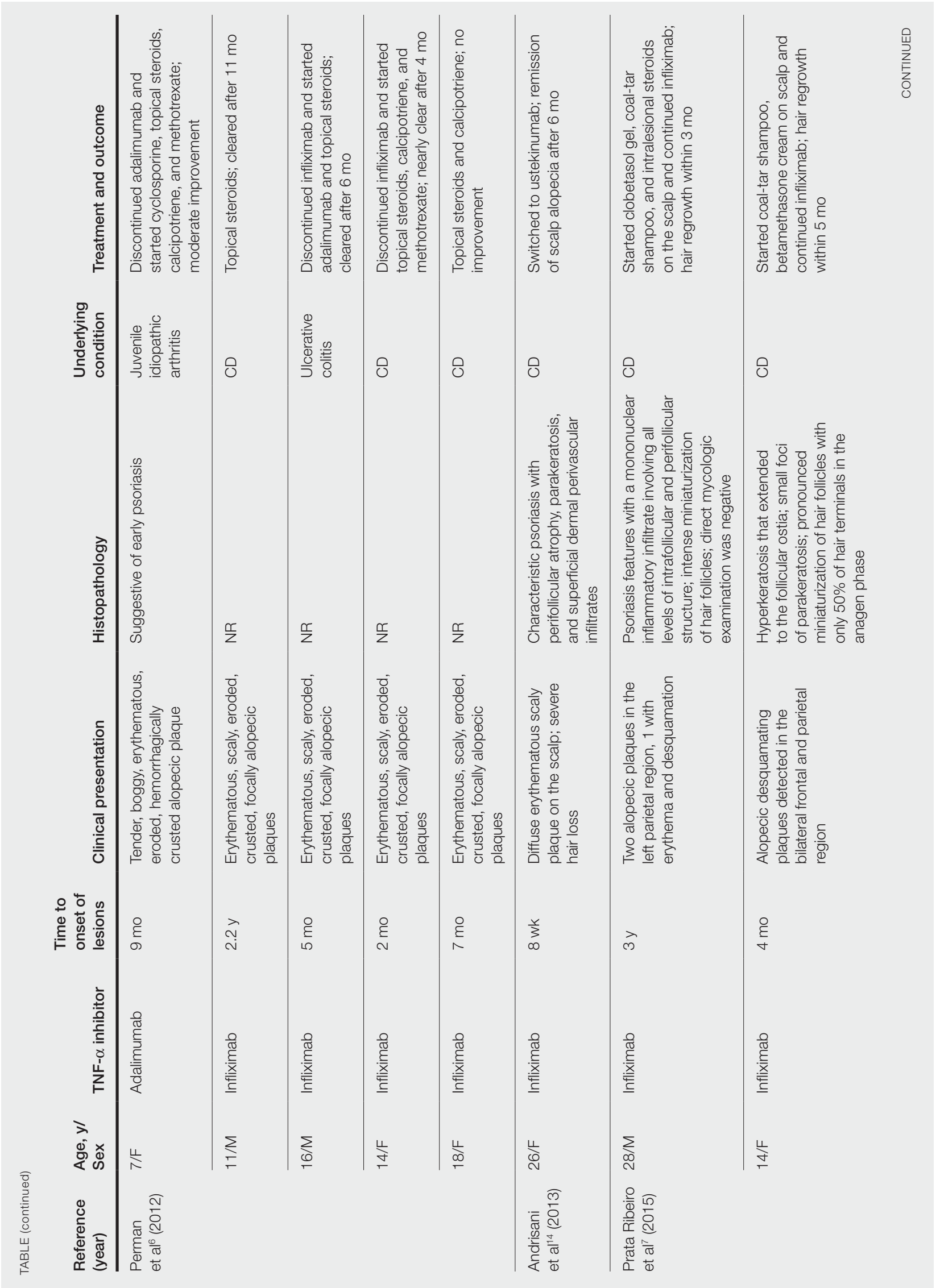




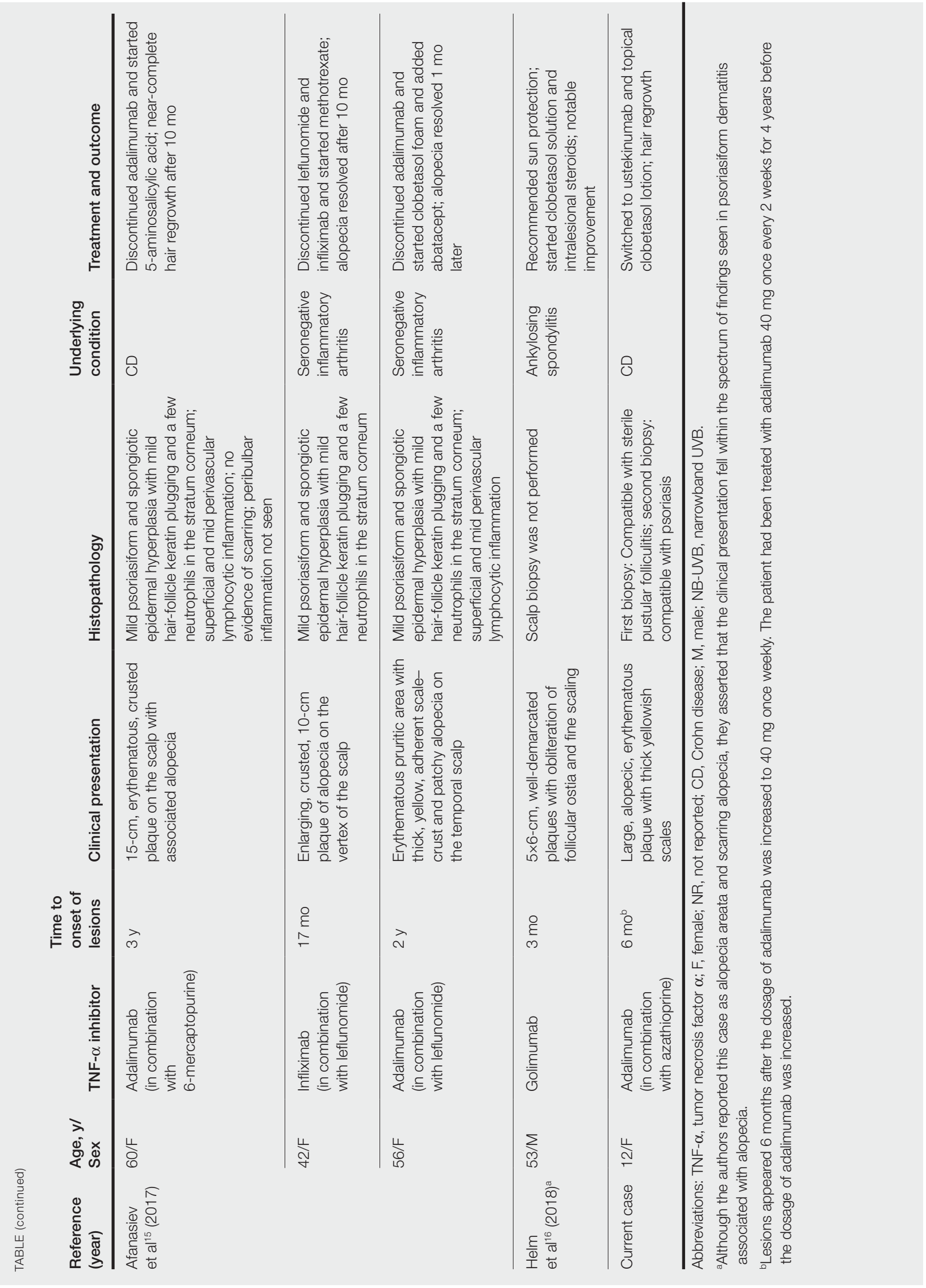




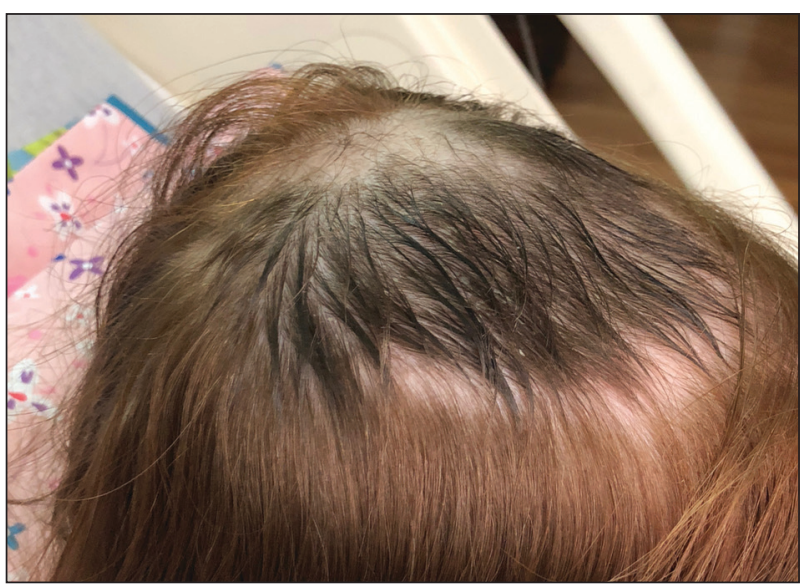

FIGURE 3. Considerable hair regrowth was noted at 6 months' follow-up.

Conditions with similar clinical findings should be ruled out before making a diagnosis of TNF- $\alpha$ inhibitor-induced psoriatic alopecia. Although clinicopathologic correlation is essential for making the diagnosis, it is possible that the histologic findings will not be specific for psoriasis. ${ }^{17}$ It is important to be aware of this condition in patients being treated with a TNF- $\alpha$ inhibitor as early as 2 months to 4 years or longer after starting treatment.

Previously reported cases have demonstrated various treatment options that yielded improvement or resolution of TNF- $\alpha$ inhibitor-induced psoriatic alopecia. These include either continuation or discontinuation of the TNF- $\alpha$ inhibitor combined with topical or intralesional steroids, methotrexate, or cyclosporine. Another option is to switch the TNF- $\alpha$ inhibitor to another biologic. Outcomes vary from patient to patient, making the physician's clinical judgment crucial in deciding which treatment route to take. Our patient showed notable improvement when she was switched from adalimumab to ustekinumab as well as the combination of ustekinumab and clobetasol lotion $0.05 \%$.

\section{Conclusion}

We recommend an individualized approach that provides patients with the safest and least invasive treatment option for TNF- $\alpha$ inhibitor-induced psoriatic alopecia. In most reported cases, the problem resolved with treatment, thereby classifying this form of alopecia as noncicatricial alopecia.

\section{REFERENCES}

1. Horneff G, Seyger MMB, Arikan D, et al. Safety of adalimumab in pediatric patients with polyarticular juvenile idiopathic arthritis, enthesitis-related arthritis, psoriasis, and Crohn's disease. J Pediatr. 2018;201:166-175.e3. doi:10.1016/j.jpeds.2018.05.042

2. Brown G, Wang E, Leon A, et al. Tumor necrosis factor- $\alpha$ inhibitorinduced psoriasis: systematic review of clinical features, histopathological findings, and management experience. J Am Acad Dermatol. 2017;76:334-341. doi:10.1016/j.jaad.2016.08.012

3. George SMC, Taylor MR, Farrant PBJ. Psoriatic alopecia. Clin Exp Dermatol. 2015;40:717-721. doi:10.1111/ced.12715

4. Shuster S. Psoriatic alopecia. Br J Dermatol. 1972;87:73-77. doi:10.1111/j.1365-2133.1972.tb05103.x

5. Silva CY, Brown KL, Kurban AK, et al. Psoriatic alopecia-fact or fiction? a clinicohistopathologic reappraisal. Indian J Dermatol Venereol Leprol. 2012;78:611-619. doi:10.4103/0378-6323.100574

6. Perman MJ, Lovell DJ, Denson LA, et al. Five cases of anti-tumor necrosis factor alpha-induced psoriasis presenting with severe scalp involvement in children. Pediatr Dermatol. 2012;29:454-459. doi:10.1111/j.1525-1470.2011.01521.x

7. Prata Ribeiro LB, Gonçalves Rego JC, Duque Estrada B, et al. Alopecia secondary to anti-tumor necrosis factor-alpha therapy. An Bras Dermatol. 2015;90:232-235. doi:10.1590/abd1806-4841.20153084

8. Papadavid E, Gazi S, Dalamaga M, et al. Palmoplantar and scalp psoriasis occurring during anti-tumour necrosis factor-alpha therapy: a case series of four patients and guidelines for management. J Eur Acad Dermatol Venereol. 2008;22:380-382. doi:10.1111/j.1468-3083.2007.02335.x

9. Manni E, Barachini P. Psoriasis induced by infliximab in a patient suffering from Crohn's disease. Int J Immunopathol Pharmacol. 2009;22:841-844. doi:10.1177/039463200902200331

10. El Shabrawi-Caelen L, La Placa M, Vincenzi C, et al. Adalimumabinduced psoriasis of the scalp with diffuse alopecia: a severe potentially irreversible cutaneous side effect of TNF-alpha blockers. Inflamm Bowel Dis. 2010;16:182-183. doi:10.1002/ibd.20954

11. Medkour F, Babai S, Chanteloup E, et al. Development of diffuse psoriasis with alopecia during treatment of Crohn's disease with infliximab. Gastroenterol Clin Biol. 2010;34:140-141. doi:10.1016/j.gcb.2009.10.021

12. Doyle LA, Sperling LC, Baksh S, et al. Psoriatic alopecia/ alopecia areata-like reactions secondary to anti-tumor necrosis factor- $\alpha$ therapy: a novel cause of noncicatricial alopecia. Am J Dermatopathol. 2011;33:161-166. doi:10.1097/DAD.0b013e3181ef7403

13. Osório F, Magro F, Lisboa C, et al. Anti-TNF-alpha induced psoriasiform eruptions with severe scalp involvement and alopecia: report of five cases and review of the literature. Dermatology. 2012;225:163-167. doi:10.1159/000342503

14. Andrisani G, Marzo M, Celleno L, et al. Development of psoriasis scalp with alopecia during treatment of Crohn's disease with infliximab and rapid response to both diseases to ustekinumab. Eur Rev Med Pharmacol Sci. 2013;17:2831-2836.

15. Afanasiev OK, Zhang CZ, Ruhoy SM. TNF-inhibitor associated psoriatic alopecia: diagnostic utility of sebaceous lobule atrophy. J Cutan Pathol. 2017;44:563-569. doi:10.1111/cup.12932

16. Helm MM, Haddad S. Alopecia areata and scarring alopecia presenting during golimumab therapy for ankylosing spondylitis. N Am J Med Sci. 2018;11:22-24. doi:10.7156/najms.2018.110122

17. Laga AC, Vleugels RA, Qureshi AA, et al. Histopathologic spectrum of psoriasiform skin reactions associated with tumor necrosis factor-a inhibitor therapy. a study of 16 biopsies. Am J Dermatopathol. 2010;32:568-573. doi:10.1097/DAD.0b013e3181cb3ff7 